Universidade Tecnológica Federal do Paraná - UTFPR

Campus Ponta Grossa - Paraná - Brasil

ISSN: 1981-3686/ v. 04, n. 01: p. 52-57, 2010

D.O.I.: $10.3895 / \mathrm{S} 1981-36862010000100006$

\author{
Revista Brasileira de Tecnologia \\ Agroindustrial
}

\title{
COMPORTAMENTO REOLÓGICO DO MEL DE Apis mellifera DO MUNICÍPIO DE TABULEIRO DO NORTE-CE
}

\section{RHEOLOGICAL BEHAVIOR OF Apis mellifera HONEY OF THE MUNICIPALITY OF TABULEIRO DO NORTE-CE}

\author{
Kelly de Fátima Nogueira Lima Silva ${ }^{1}$; Dyego da Costa Santos ${ }^{1}$; Charles Teles Santos da Silva ${ }^{1}$; \\ Alexandre José de Melo Queiroz ${ }^{2}$ Afonso Odério Nogueira Lima ${ }^{3}$ \\ ${ }^{1}$ Faculdade de Tecnologia - CENTEC - Limoeiro do Norte - Brasil fafa charles13@ yahoo.com.br \\ ${ }^{2}$ Universidade Federal de Campina Grande - UFCG - Brasil charles_fafa@ yahoo.com.br \\ ${ }^{3}$ Universidade Federal do Ceará - UFC - Brasil oderio@ bisanet.com.br
}

\begin{abstract}
Resumo
O objetivo do trabalho foi estudar as características de viscosidade dos méis do Município de Tabuleiro do Norte-CE nas temperaturas de 20, 25, 30, 35 e 40 ${ }^{\circ} \mathrm{C}$ em função do tempo de armazenamento de 0, 45 e 90 dias. Os méis utilizados foram obtidos a partir de coletas realizadas por apicultores de Tabuleiro do Norte-CE e conduzidos ao Laboratório de Processamento e Armazenamento de Produtos Agrícolas da UFCG, onde o estudo foi conduzido. Para determinação das viscosidades foi utilizado um viscosímetro da marca Brookfield, modelo RVT. Verificou-se que o armazenamento não provocou aumentos ou reduções conseqüentes de viscosidade, no entanto o aumento de temperatura resultou em diminuições de viscosidade, com diferenças significativas entre todas as amostras. Diante dos resultados conclui-se que com o aumento da temperatura, ocorre uma redução na viscosidade dos méis, sendo mais acentuada com o início do aquecimento, entre 20 e $30^{\circ} \mathrm{C}$.
\end{abstract}

Palavras-chave: mel, viscosidade, armazenamento.

\section{Introdução}

Segundo Kramer (1966), viscosidade pode ser definida como a fricção de um fluido, isto é, a resistência encontrada pelas moléculas em se mover no interior do fluido devido ao movimento browniano e as forças intermoleculares. O termo viscosidade é aplicado apenas quando se trata de fluidos quimicamente puros e fisicamente homogêneos (fluidos newtonianos) enquanto o termo consistência é comumente aplicado a produtos alimentícios, os quais não são quimicamente puros nem fisicamente homogêneos (fluidos não-newtonianos). No caso do mel, a viscosidade é identificada, pelo consumidor, como uma característica intrínseca, servindo como parâmetro de determinação de 
qualidade e de preferência. Uma caracterização rigorosa das faixas de viscosidade apresentadas pelos méis de uma determinada origem se constitui numa referência importante para a valorização do seu conjunto de atributos, justificando o seu estudo inclusive sob efeitos de aquecimento.

Alguns méis apresentam particularidades ou anomalias em relação à sua viscosidade (Crane, 1983; Campos, 1987). No Piauí é freqüente os méis produzidos no primeiro semestre apresentarem-se mais fluidos que aqueles coletados no segundo semestre, particularmente em anos de chuvas abundantes. A umidade do ar, bem como a precipitação pluviométrica, ou ainda particularidades de cada florada, são provavelmente as responsáveis por essas características (Freitas, 1994).

O objetivo do trabalho foi estudar as características viscosas dos méis do Município de Tabuleiro do Norte-CE nas temperaturas de 20, 25, 30, 35 e $40{ }^{\circ} \mathrm{C}$ em função do tempo de armazenamento de 0,45 e 90 dias.

\section{Material e métodos}

Obtenção do mel

Os méis utilizados neste trabalho, produzidos por Apis mellífera L. com predominância da florada de vassourinha de botão, foram obtidos no mês de junho de 2004, a partir de coletas realizadas por apicultores no município de Tabuleiro do Norte, localizado na Região do Baixo Jaguaribe - CE.

As melgueiras, ao chegarem à casa de mel, foram colocadas sobre bandejas de aço inoxidável e levadas para a sala de recepção dos favos; em seguida foram depositadas em área isolada do recinto, onde ocorreu a extração do mel e as outras etapas do processamento.

Após a desoperculação dos favos, os quadros foram encaminhados para a centrífuga, onde se iniciou lentamente o processo, aumentando-se a sua velocidade progressivamente para não quebrar os quadros que estão cheios de mel. Uma vez extraído, o mel foi retirado da centrífuga por um sistema de bombeamento, escoando-o diretamente para o decantador iniciando-se assim o processo de filtragem. Após a filtragem, o mel foi encaminhado para o decantador, onde "descansou" por 48 horas, a fim de que as eventuais partículas que não foram retiradas pela filtragem e as bolhas criadas durante o processo se deslocassem para a porção superior do decantador, onde foram retiradas posteriormente durante o procedimento de envase.

Os méis do município de Tabuleiro do Norte-CE foram acondicionados em recipientes de vidro, envoltos em papel alumínio e conduzidos ao Laboratório de Processamento Armazenamento e de Produtos Agrícolas da Universidade Federal de Campina Grande-PB onde foram realizados os ensaios para a determinação das viscosidades. 


\section{Método de Análise}

Para a determinação das viscosidades dos méis do município de Tabuleiro do Norte-CE foi utilizado um viscosímetro da marca Brookfield, modelo RVT. Foram realizadas leituras de torque nas velocidades de rotação de $50 \mathrm{rpm}$, nas temperaturas de 20, 25, 30, 35 e $40^{\circ} \mathrm{C}$. As viscosidades dos méis foram determinadas logo após o processamento (tempo inicial 0) e aos 45 e 90 dias.

Para avaliar o efeito da temperatura sobre as viscosidades dos méis, foram feitas regressões polinomiais dos dados utilizando-se o software Origin, versão 7.5. O coeficiente de determinação $\left(\mathrm{R}^{2}\right)$ foi utilizado como medida da adequação do ajuste das equações às medidas experimentais.

Também foi utilizada a equação clássica de Arrhenius para avaliar o efeito da temperatura sobre as viscosidades.

\section{Resultados e Discussão}

A análise de variância para os valores das viscosidades do mel de Tabuleiro do Norte encontrase na Tabela 1, onde se observa, através do teste F, que existe diferença significativa ao nível de $1 \%$ de probabilidade nos fatores tempo, temperatura e na interação dos fatores.

Tabela 1 - Análise de variância das viscosidades (Pa.s) para méis do município de Tabuleiro, com florada da vassourinha de botão, submetido a três meses de armazenamento em diferentes temperaturas

\begin{tabular}{lcccc}
\hline \multirow{2}{*}{ Fonte de variação } & \multicolumn{5}{c}{ Análise de variância } \\
\cline { 2 - 5 } & G.L & S.Q & Q.M & F \\
\hline Tempo & 2 & 9,33192 & 4,66596 & $769541591210663 * *$ \\
Temperatura & 4 & 1414,55416 & 353,63854 & $5,83244530139592^{* * *}$ \\
Tempo X Temp. & 8 & 41,52848 & 5,19106 & $856144624572403 * *$ \\
Resíduo & 75 & 0,00000 & 0,00000 & \\
\hline Total & 89 & 1465,41456 & & \\
\hline
\end{tabular}

Na Tabela 2 são apresentadas as viscosidades do mel de Tabuleiro do Norte armazenado por 90 dias entre 20 e $40^{\circ} \mathrm{C}$. O valor médio da viscosidade obtido nos ensaios foi de 4,276 Pa.s para um intervalo de variação de 14,0 Pa.s a 0,78 Pa.s.

Tabela 2 - Valores médios das viscosidades (Pa.s) para méis do município de Tabuleiro do Norte, com florada da vassourinha de botão. Interação tempo de armazenamento x temperatura

\begin{tabular}{cccccc}
\hline Tempo de & \multicolumn{5}{c}{ Temperatura $\left({ }^{\circ} \mathbf{C}\right)$} \\
\cline { 2 - 6 } armazenamento (dia) & $\mathbf{2 0}$ & $\mathbf{2 5}$ & $\mathbf{3 0}$ & $\mathbf{3 5}$ & $\mathbf{4 0}$ \\
\hline 0 & $10,2000 \mathrm{cA}$ & $4,9000 \mathrm{aB}$ & $2,5200 \mathrm{bC}$ & $1,7200 \mathrm{aD}$ & $1,1500 \mathrm{aE}$ \\
45 & $11,2000 \mathrm{bA}$ & $4,5300 \mathrm{cB}$ & $1,7600 \mathrm{cC}$ & $1,5700 \mathrm{bD}$ & $0,9500 \mathrm{bE}$ \\
90 & $14,0000 \mathrm{aA}$ & $4,5600 \mathrm{bB}$ & $2,7600 \mathrm{aC}$ & $1,5400 \mathrm{cD}$ & $0,7800 \mathrm{cE}$ \\
\hline
\end{tabular}


DMS p/ colunas = 0,0000; DMS p/ linhas $=0,0000 ; \mathrm{MG}=4,2760 \mathrm{~Pa} . \mathrm{S} ; \mathrm{CV}=0,00 \%$. DMS: Desvio mínimo significativo; MG: Média geral e CV: Coeficiente de variação. Obs.: médias seguidas pela mesma letra minúscula nas colunas e maiúsculas nas linhas, não diferem estatisticamente pelo teste de Tukey, a 5\% de probabilidade.

Verifica-se que o armazenamento não provocou aumentos ou reduções conseqüentes de viscosidade. Percebe-se que as maiores viscosidades se encontram em dois tempos, tempo zero nas temperaturas de 25,35 e $40{ }^{\circ} \mathrm{C}$ e tempo de 90 dias para as temperaturas de 20 e $30{ }^{\circ} \mathrm{C}$. O aumento de temperatura resultou em diminuições de viscosidade, com diferenças significativas entre todas as amostras.

O mel de Tabuleiro do Norte apresentou uma variação na viscosidade de $89 \%, 91 \%$ e $94 \%$ nos tempos de zero, 45 e 90 dias de armazenamento, respectivamente, entre as temperaturas de 20 a $40{ }^{\circ} \mathrm{C}$. Essas variações foram próximas às encontradas por Silva (2001), que, analisando a viscosidade do mel da florada silvestre do Estado do Piauí, obteve uma variação de 92\%. Sodré et al. (2002) encontraram uma média de 1,607 Pa.s para viscosidade nos méis provenientes dos Estados do Ceará, Piauí e Pernambuco.

Apresenta-se, na Figura 1, o gráfico do comportamento das viscosidades do mel do município de Tabuleiro do Norte durante o armazenamento. Observa-se que nas temperaturas de 25,35 e $40{ }^{\circ} \mathrm{C}$ os pontos se confundem; na temperatura de $20^{\circ} \mathrm{C}$ estes se apresentam dispersos e na temperatura de $30{ }^{\circ} \mathrm{C}$ os valores nos tempos 0 e 90 dias se aproximam, o que não ocorre com o tempo de 45 dias que se afasta dos demais. Este comportamento irregular refletiu a ausência de efeito do tempo de armazenamento sobre as viscosidades.

Figura 1 - Viscosidades aparentes (Pa.s), do mel de Tabuleiro do Norte produzido a partir da florada de vassourinha de botão, em função da temperatura nos diferentes tempos de armazenamento

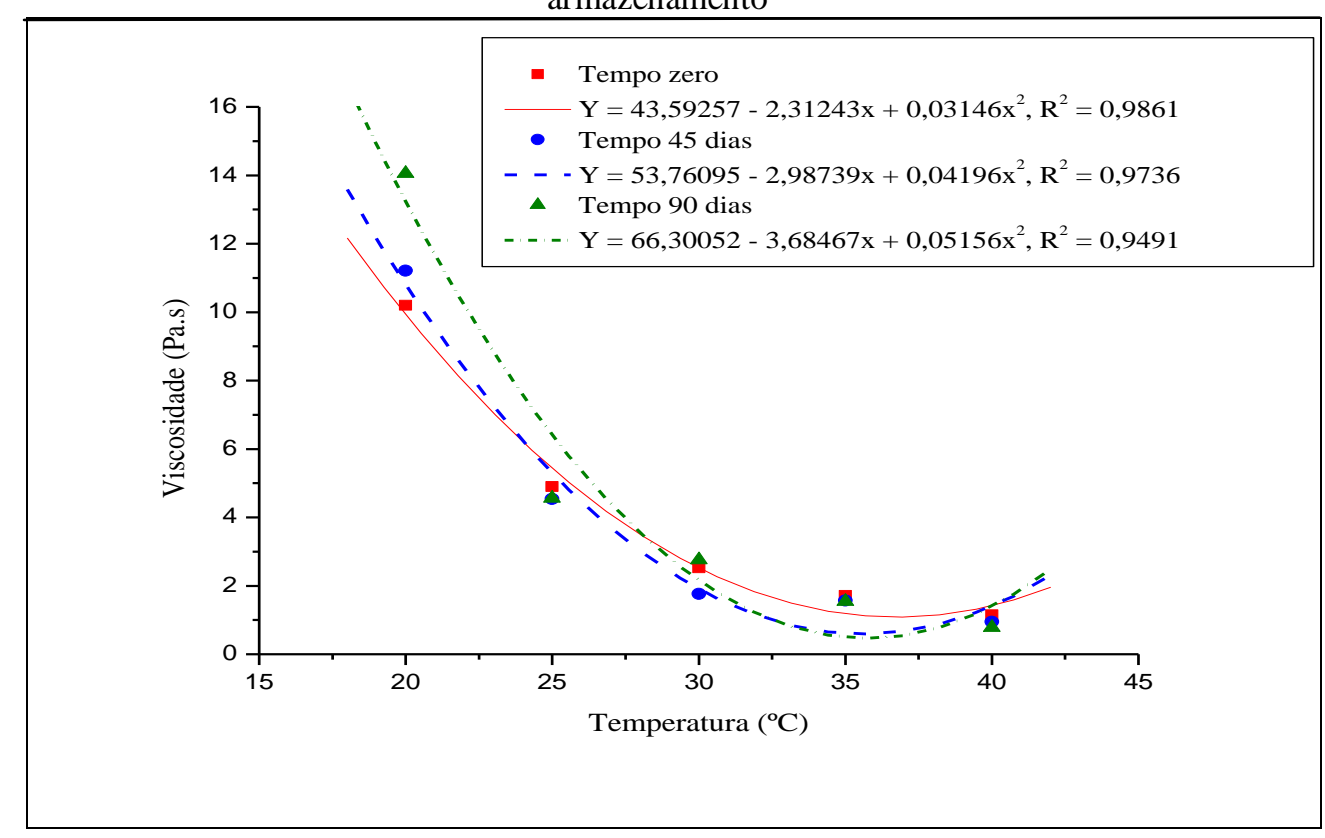


O coeficiente de determinação $\left(\mathrm{R}^{2}\right)$ variou de 0,9491 para o tempo 90 dias e 0,9861 para o tempo de zero dia de armazenamento, configurando um bom ajuste aos dados de viscosidade calculados, podendo as equações quadráticas ser utilizadas para estimar a viscosidade em função da temperatura.

$\mathrm{Na}$ representação gráfica da Figura 1, tem-se o comportamento das viscosidades nas temperaturas de 20 a $40^{\circ} \mathrm{C}$ dos méis de Tabuleiro do Norte-CE, onde são apresentados os pontos experimentais obtidos no tempo zero (zero dia), tempo 1 (45 dias) e tempo 2 (90 dias). As curvas, referentes aos três conjuntos de pontos, foram obtidas por ajustes com equação quadrática, que resultou em bons coeficientes de determinação. Em todos os casos as viscosidades apresentaram reduções mais acentuadas com o início do aquecimento, entre 20 e $30^{\circ} \mathrm{C}$, explicando a boa interpretação da relação pelo tipo de ajuste utilizado. Na maioria dos casos a alternância de posição entre as curvas demonstra que o tempo de armazenamento não influenciou as viscosidades das amostras estudadas.

A redução da viscosidade com o aumento da temperatura é um comportamento comum com os fluidos alimentícios, também observados por Silva (2001) em méis de abelha (Apis mellifera L.) produzidos no Estado do Piauí, e por Junzheng \& Changying (1998) em vários méis da China.

Yanniotis et al. (2004), estudando o efeito da umidade na viscosidade do mel de várias floradas em diferentes temperaturas, constataram que a viscosidade do mel varia com a temperatura, umidade e sua origem botânica.

\title{
4. Conclusão
}

Com o aumento da temperatura, ocorreu uma redução na viscosidade dos méis, sendo mais acentuada com o início do aquecimento, entre 20 e $30^{\circ} \mathrm{C}$. A equação de Arrhenius representou adequadamente o efeito da temperatura na viscosidade do mel, nos três tempos de armazenamento estudados.

\begin{abstract}
The objective was to study the characteristics of viscous honey of Tabuleiro do Norte-CE at 20, 25, 30, 35 and $40^{\circ} \mathrm{C}$ depending on the storage time of 0,45 and 90 days. The honeys used were obtained from collections made by beekeepers Board North-EC and led to the Laboratory Handling and Storage of Agricultural Products UFCG, where the study was conducted. To determine the viscosity was used to mark a Brookfield viscometer, model RVT. It was found that the store did not cause increases or reductions resulting viscosity, however the increase in temperature resulted in decreases in viscosity, with significant differences between all samples. From the results it is concluded that with increasing
\end{abstract}


temperature, there is a reduction in the viscosity of honey was more pronounced with the onset of the heating, between 20 and $30^{\circ} \mathrm{C}$.

Key-words: honey, viscosity, storage.

\section{Referências}

CAMPOS, M. da G. R. Contribuição para o estudo do mel, pólen, geléia real e própolis. Boletim da Faculdade de Farmácia de Coimbra, Coimbra, v. 11, n. 2, p. 17-47, 1987.

CRANE, E. O Livro do mel. São Paulo: Nobel, 1983. 226 p.

FREITAS, B.M. Beekeping and cashew in north-eastern Brazil: the balance of honey and nut production. Bee World, Cardiff, v. 75, n. 4, p. 168-177, 1994.

JUNZHENG, P.; CHANGYING, J. General rheological model for natural honeys in China, Journal of Food Engineering, Essex, v. 36, n. 2, p. 165-168, 1998.

KRAMER, A.; TWIGG, B. A. Fundamentals of quality control for the food industry. $2^{\mathrm{a}}$ ed.Westport: AVI, 1966. 541 p.

SILVA, C. L. Caracterização reológica e físico-química de méis de abelha (Apis mellifera L.) do estado do Piauí. 2001. 87 f. Dissertação (Mestrado em Engenharia Agrícola) - Universidade Federal de Campina Grande, Campina Grande.

SODRÉ, G. DA S.; MARCHINI, L. C.; ARRUDA, C. M. F.; LEVY, P. S. Viscosidade e umidade de amostras de méis de Apis mellifera de estados da região Nordeste do Brasil. In: SIMPOSIO INTERNACIONAL DE INICIAÇÃO CIENTIFICA DA UNIVERSIDADE DE SÃO PAULO, 10., Piracicaba, 2002. Anais. Piracicaba: Universidade de São Paulo, 2002. CD.

YANNIOTIS. S.; SKALTSI, S.; KARABURNIOTI, S.. Effect of moisture content on the viscosity of honey at different temperatures. Journal of Food Engineering. Food Science and Technology Department, Agricultural University of Athens, Iera Odos 75, Athens 11855, Greece, 21 dec. 2004. p. $1-6$.

Dados do Autor para Correspondência:

Nome: Kelly de Fátima Nogueira Lima Silva

Filiação institucional: Faculdade de Tecnologia CENTEC - Limoeiro do Norte

Departamento: Tecnologia de Alimentos

Função ou cargo ocupado: Professora

Titulação: Mestre em Engenharia Agrícola - UFCG

Endereço: Avenida Dom Aureliano Matos, 1007, Apto. 102. Centro, Limoeiro do Norte-CE, Brasil. CEP: 62930-000

Telefones para contato: (88) 99217613

e-mail: fafa_charles13@yahoo.com.br 